\title{
GENERALIZED FOKKER-PLANCK EQUATIONS AND CONVERGENCE TO THEIR EQUILIBRIA
}

\author{
PIOTR BILER and GRZEGORZ KARCH \\ Instytut Matematyczny, Uniwersytet Wroclawski \\ pl. Grunwaldzki 2/4, 50-384 Wroctaw, Poland \\ E-mail: Piotr.Biler@math.uni.wroc.pl,Grzegorz.Karch@math.uni.wroc.pl \\ Institute of Mathematics of the Polish Academy of Sciences (2002-2003)
}

In memory of Adam Rybarski (1930-2001)

\begin{abstract}
We consider extensions of the classical Fokker-Planck equation $u_{t}+\mathcal{L} u=$ $\nabla \cdot(u \nabla V(x))$ on $\mathbb{R}^{d}$ with $\mathcal{L}=-\Delta$ and $V(x)=\frac{1}{2}|x|^{2}$, where $\mathcal{L}$ is a general operator describing the diffusion and $V$ is a suitable potential.
\end{abstract}

1. Introduction. We consider the initial value problem for the generalized FokkerPlanck equation

$$
\begin{aligned}
u_{t}+\mathcal{L} u & =\nabla \cdot(u \nabla V), \quad x \in \mathbb{R}^{d}, \quad t>0, \\
u(x, 0) & =u_{0}(x) \in L^{1}\left(\mathbb{R}^{d}\right),
\end{aligned}
$$

with a sufficiently regular potential $V=V(x), u=u(x, t)$ and $u_{0}(x) \geq 0$. This equation generalizes the classical Fokker-Planck equation

$$
u_{t}-\Delta u=\nabla \cdot(u x)
$$

in two ways. First, the second order elliptic differential operator $-\Delta$ is replaced by a Markov diffusion operator $\mathcal{L}$ so that $-\mathcal{L}$ generates a positivity and mass preserving semigroup $e^{-t \mathcal{L}}$ on $L^{1}\left(\mathbb{R}^{d}\right)$. Second, the potential $V(x)=\frac{1}{2}|x|^{2}$ with $\nabla V(x)=x$ is replaced by a more general potential $V$ which is large enough as $|x| \rightarrow \infty$ so that $V$ is confining.

Our assumptions below will guarantee the existence of the unique steady state $u_{\infty}=$

2000 Mathematics Subject Classification: Primary 35K90; Secondary 35B40.

Key words and phrases: Fokker-Planck equation, steady states, asymptotics of solutions, relative entropy.

The paper is in final form and no version of it will be published elsewhere. 
$u_{\infty}(x) \geq 0$ of (1.1) with given $M>0$,

$$
\int u_{\infty}(x) d x=\int u_{0}(x) d x \equiv M
$$

Of course, the Gaussian function $u_{\infty}(x)=M(2 \pi)^{-d / 2} \exp \left(-|x|^{2} / 2\right)$ is the steady state for (1.3) for each $M>0$, and $u(t)$ tends to $u_{\infty}$ at an exponential rate in all the $L^{p}$ norms, $1 \leq p \leq \infty$, cf. [21].

The motivations to study extensions (1.1) of (1.3) stem from the probability theory where Fokker-Planck equations are deeply connected with (nonlinear) stochastic differential equations driven by Gaussian and Lévy processes, see e.g. [17]. Another motivation is a study of the large time behavior of solutions of linear (as well as nonlinear) equations

$$
v_{t}+\mathcal{L} v=0
$$

with general diffusion operators $\mathcal{L}$. Here, suitable space-time rescaling leads to an equation of type (1.1) with $V(x)=\frac{1}{2}|x|^{2}$ and $\mathcal{L}$ enjoying some invariance properties (like generators of symmetric $\alpha$-stable processes), see [8] and [6], [7], [9] for various results on this subject and its modifications. Main results include self-similar asymptotics of solutions of (1.5) when $t \rightarrow \infty$, which was obtained for the model problem $u_{t}-\Delta u=0$ already in [19]. Physical motivations to study (1.5) and (1.1) come from analysis of anomalous diffusion phenomena, see introductions in [7], [8] for more details.

Nonlinear versions of Fokker-Planck equations appear e.g. in statistical mechanics, where the determination of the decay rate of solutions to the (thermal) equilibrium state is an important problem, see [1, Sec. 4], [2], [4], [3], etc. Let us also mention the paper [18], where different nonlinear Fokker-Planck equations have been studied using entropy methods.

The recent paper [1] deals with the models (1.1) with classical diffusion given by a divergence form second order elliptic differential operator $\mathcal{L}$ (with variable coefficients). The study of the trend to equilibrium is based on a systematic use of various entropy functionals and Csiszár-Kullback inequalities relating entropies to usual $L^{p}$ norms, see for details [22]. The exponential decay in time of the entropy is derived using the equations for entropy and entropy dissipation and convex Sobolev inequalities (Sec. 3 in [1]) generalizing the classical logarithmic Sobolev inequality of L. Gross (see, e.g. [21] and [1] for various approaches to this subject) as well as Poincaré type inequalities.

Our goal in this paper is to give simple entropy dissipation arguments which could be the first step toward proving that solutions approach steady states in $L^{1}$ for fairly general equations (1.1). Next, by the Fourier analysis, we show the exponential decay of $\left\|u(t)-u_{\infty}\right\|_{L^{p}}$ for equations (1.1) with a Lévy diffusion generator $\mathcal{L}$ close to a fractional power of the Laplacian operator $(-\Delta)^{\alpha / 2}, 0<\alpha<2$, and $V(x)=\frac{1}{2}|x|^{2}$. We will also briefly comment on the difficulties with the application of logarithmic Sobolev inequalities for generators of symmetric Markov semigroups (as in [13]) to the problem of the optimal decay rate of the entropy when $\mathcal{L}$ is not a (divergence form) differential operator.

Notation. All the integrals without integration limits are meant $\int_{\mathbb{R}^{d}} \ldots$; inessential constants are denoted by $C$, even if they vary from line to line. 


\section{Entropy dissipation for solutions of general Fokker-Planck equations.} We prove in this section the decreasing property of the (relative with respect to the steady states) entropy of solutions $u$ of the Fokker-Planck equation (1.1), under fairly general assumptions on $\mathcal{L}$ and $V$. Namely, we suppose that the operator $\mathcal{L}$ is Markov, i.e. $-\mathcal{L}$ generates a positivity and mass preserving strongly continuous semigroup $\left\{e^{-t \mathcal{L}}\right\}_{t \geq 0}$ on $L^{1}\left(\mathbb{R}^{d}\right)$, and suppose that $e^{-t \mathcal{L}}$ is defined by an integral kernel $K_{t}$ :

$$
e^{-t \mathcal{L}} z(x)=\int K_{t}(x, y) z(y) d y
$$

The latter follows, e.g., from the ultracontractivity assumption

$$
e^{-t \mathcal{L}}: L^{1}\left(\mathbb{R}^{d}\right) \rightarrow L^{\infty}\left(\mathbb{R}^{d}\right), \quad t>0,
$$

by the Dunford-Pettis theorem, see [15, Th. 2.6.20].

We will not use in this section explicit characterizations of $\mathcal{L}$ which can be given in the form of pseudodifferential operators or integro-differential ones, cf. [15, Sec. 3.7].

Moreover, we assume that $V$ is a confinement potential with respect to $\mathcal{L}$, i.e. the steady states $u_{\infty}$ satisfying the equation

$$
\mathcal{L} u_{\infty}=\nabla \cdot\left(u_{\infty} \nabla V\right)
$$

are positive: $u_{\infty}(x) \geq 0$, integrable: $u_{\infty} \in L^{1}\left(\mathbb{R}^{d}\right)$, and normalized: $\int u_{\infty}(x) d x=1$. Recall that for $\mathcal{L}=-\Delta$ this integrability condition is equivalent to $e^{-V} \in L^{1}\left(\mathbb{R}^{d}\right)$, while $V \in W_{\text {loc }}^{2, \infty}\left(\mathbb{R}^{d}\right)$ is assumed to have $u_{\infty}$ regular enough. We will see in the next section how to solve $(2.2)$ if $\mathcal{L}$ is a Lévy operator defined by a Fourier multiplier $a(\xi)$ : $\widehat{\mathcal{L} u(\xi)}=a(\xi) \widehat{u}(\xi)$, and $V(x)=\frac{1}{2}|x|^{2}$. In those cases the steady states $u_{\infty}$ are unique up to the normalization $\int u_{\infty}(x) d x=1$.

We consider relative entropy functionals of the form

$$
W\left[u(t) \mid u_{\infty}\right] \equiv W(t)=\int \Psi\left(\frac{u(x, t)}{u_{\infty}(x)}\right) u_{\infty}(x) d x,
$$

where $u(t)=u(x, t) \geq 0$ is a solution of (1.1), the function $\Psi$ is a $C^{2}$ convex function on $\mathbb{R}^{+}, \Psi(s) \geq 0$ for $s \geq 0$, and $\Psi(1)=0$. Typical (and the most important for our purposes) examples include the logarithmic (or physical) entropy with

$$
\Psi_{1}(s)=s \log s-s+1,
$$

and the quadratic entropy

$$
\Psi_{2}(s)=(s-1)^{2} .
$$

More generally, one can consider the family of entropies generated by

$$
\Psi_{p}(s)=s^{p}-1-p(s-1)
$$

with $1<p \leq 2$, the logarithmic entropy being obtained in the limit as $p \searrow 1$. They have been considered in [1] as examples of admissible entropies. However, in this section we need neither additional regularity assumptions on $\Psi$ (like $\Psi \in C^{4}$ ), nor growth restrictions (like $\Psi(s) \leq C(s-1)^{2}$ ) used in [1] in order to consider not only the entropy production equation (2.8) below but also the rate of entropy dissipation, see (2.15), below. 
THEOREM 2.1. Suppose that $u=u(x, t) \geq 0$ is a sufficiently regular solution of the Fokker-Planck equation (1.1), $\Psi$ generates an entropy functional as above, and $u_{0} \in$ $L^{1}\left(\mathbb{R}^{d}\right)$ satisfies moreover $W\left[u_{0} \mid u_{\infty}\right]<\infty$ for a steady state $u_{\infty}$ with $\int u_{\infty}=\int u_{0}$. Then the entropy $W$ decreases monotonically: $W(t) \searrow W_{\infty}$ as $t \rightarrow \infty$.

PROOF. We begin with formal calculations valid for regular solutions, noting that for $u_{0}$ admitting a solution of the initial value problem (1.1)-(1.2) and satisfying merely the condition $W\left[u_{0} \mid u_{\infty}\right]<\infty$, the calculations can be justified by standard approximation procedures as in, e.g., [1].

Differentiating the entropy functional $W$ along the solution $u(t)$ of (1.1) we get after some integrations by parts

$$
\begin{aligned}
\frac{d W}{d t} & =\int \Psi^{\prime}\left(\frac{u(x, t)}{u_{\infty}(x)}\right) u_{t}(x, t) d x \\
& =-\int \mathcal{L} u \Psi^{\prime}\left(\frac{u}{u_{\infty}}\right)+\int \nabla \cdot(u \nabla V) \Psi^{\prime}\left(\frac{u}{u_{\infty}}\right) \\
& =-\int \mathcal{L} u \Psi^{\prime}\left(\frac{u}{u_{\infty}}\right)-\int u \nabla V \Psi^{\prime \prime}\left(\frac{u}{u_{\infty}}\right) \cdot \nabla\left(\frac{u}{u_{\infty}}\right) \\
& =-\int \mathcal{L} u \Psi^{\prime}\left(\frac{u}{u_{\infty}}\right)-\int \nabla V u_{\infty} \cdot \nabla \varphi\left(\frac{u}{u_{\infty}}\right)
\end{aligned}
$$

where $\varphi^{\prime}(s)=s \Psi^{\prime \prime}(s)$ so that

$$
\varphi(s)=s \Psi^{\prime}(s)-\Psi(s) .
$$

Finally, we get the entropy production formula

$$
\frac{d W}{d t}=-\int \mathcal{L} u \Psi^{\prime}\left(\frac{u}{u_{\infty}}\right)+\int \mathcal{L} u_{\infty} \varphi\left(\frac{u}{u_{\infty}}\right) \equiv-\mathcal{I}
$$

since the steady state satisfies the relation (2.2).

The integration by parts was used systematically in [1] which permitted the authors to transform the integral $\int \mathcal{L} u \Psi^{\prime}\left(\frac{u}{u_{\infty}}\right)$ into a more convenient expression for divergence form second order elliptic differential operators $\mathcal{L}$. Here, we no longer may use integrations by parts, hence we approximate the operator $\mathcal{L}$ by the operators $\frac{1}{h}\left(I-e^{-h \mathcal{L}}\right)$ strongly converging to $\mathcal{L}$ as $h \searrow 0$.

Thus, the right hand side $-\mathcal{I}$ of $(2.8)$ can be viewed as the limit as $h \searrow 0$ of the expressions

$$
\begin{aligned}
-\mathcal{I}_{h} \equiv & -\frac{1}{h} \int\left(I-e^{-h \mathcal{L}}\right) u \Psi^{\prime}\left(\frac{u}{u_{\infty}}\right)+\frac{1}{h} \int\left(I-e^{-h \mathcal{L}}\right) u_{\infty} \varphi\left(\frac{u}{u_{\infty}}\right) \\
= & -\frac{1}{h} \int\left\{\frac{u(y, t)}{u_{\infty}(y)} \Psi^{\prime}\left(\frac{u(y, t)}{u_{\infty}(y)}\right)-\varphi\left(\frac{u(y, t)}{u_{\infty}(y)}\right)\right\} u_{\infty}(y) d y \\
& -\frac{1}{h} \iint K_{h}(x, y)\left\{-\frac{u(y, t)}{u_{\infty}(y)} \Psi^{\prime}\left(\frac{u(x, t)}{u_{\infty}(x)}\right)+\varphi\left(\frac{u(x, t)}{u_{\infty}(x)}\right)\right\} u_{\infty}(y) d y d x
\end{aligned}
$$

where $K_{h}(\cdot, \cdot)$ denotes the integral kernel of the semigroup $e^{-h \mathcal{L}}$, cf. (2.1). In particular, $\int K_{h}(x, y) d x=1$ holds for all $h>0$ and $y \in \mathbb{R}^{d}$. 
Next, we have by (2.7) and (2.9)

$$
\begin{aligned}
\mathcal{I}_{h}=\frac{1}{h} \iint K_{h}(x, y)\left\{-\frac{u(y, t)}{u_{\infty}(y)}\right. & \Psi^{\prime}\left(\frac{u(x, t)}{u_{\infty}(x)}\right) \\
& \left.+\varphi\left(\frac{u(x, t)}{u_{\infty}(x)}\right)+\Psi\left(\frac{u(y, t)}{u_{\infty}(y)}\right)\right\} u_{\infty}(y) d y d x .
\end{aligned}
$$

Taking into account again the relation (2.7) we arrive at the representation

$$
\begin{aligned}
\mathcal{I}_{h}=\frac{1}{h} \iint K_{h}(x, y)\{\Psi( & \left.\frac{u(y, t)}{u_{\infty}(y)}\right)-\Psi\left(\frac{u(x, t)}{u_{\infty}(x)}\right) \\
& \left.+\Psi^{\prime}\left(\frac{u(x, t)}{u_{\infty}(x)}\right)\left(\frac{u(x, t)}{u_{\infty}(x)}-\frac{u(y, t)}{u_{\infty}(y)}\right)\right\} u_{\infty}(y) d y d x .
\end{aligned}
$$

Finally, $\mathcal{I}_{h}$ is positive by the convexity of $\Psi$. For instance, using the Taylor formula, the expression in braces in $(2.10)$ is equal to

$$
\frac{1}{2} \Psi^{\prime \prime}(\zeta)\left(\frac{u(x, t)}{u_{\infty}(x)}-\frac{u(y, t)}{u_{\infty}(y)}\right)^{2} \geq 0,
$$

with an intermediate point $\zeta$ between $\frac{u(x, t)}{u_{\infty}(x)}$ and $\frac{u(y, t)}{u_{\infty}(y)}$.

Remark that the positivity of $\mathcal{I}_{h}$ might be also concluded using the Young inequality

$$
a b \leq \Psi(a)+\Phi(b), \quad a, b \geq 0,
$$

where the convex function $\Phi$ is the conjugate function of $\Psi$, cf. [16], satisfying the relation $\Phi\left(\Psi^{\prime}(s)\right) \equiv \varphi(s)$, and putting $a=\frac{u(y, t)}{u_{\infty}(y)}, b=\Psi^{\prime}\left(\frac{u(x, t)}{u_{\infty}(x)}\right)$ in $(2.12)$. Remember that $\Psi^{\prime}$ and $\Phi^{\prime}$ are increasing, mutually inverse functions, and the relation $a \Psi^{\prime}(a)=\Psi(a)+\Phi\left(\Psi^{\prime}(a)\right)$ is satisfied identically by (2.7).

The differential inequality $\frac{d W}{d t} \leq 0$ following from (2.8), (2.10) and (2.11) shows that $W\left[u(t) \mid u_{\infty}\right]$ decreases along the trajectories of (1.1).

REMARK. Of course, if the uniqueness of steady states satisfying (1.4) is supposed, it is expected that $\lim _{t \rightarrow \infty} W(t)=W_{\infty}$ equals $W_{\infty}=W\left[u_{\infty} \mid u_{\infty}\right]=0$. This would imply, using the extensions of the Csiszár-Kullback inequalities studied in [22, Sec. 2.7]

$$
\left\|u(t)-u_{\infty}\right\|_{L^{1}} \leq K W\left[u(t) \mid u_{\infty}\right]
$$

for admissible entropies $W$ and some constant $K=K(\Psi)$, that $u(t)$ converges to $u_{\infty}$ in $L^{1}\left(\mathbb{R}^{d}\right)$. We cannot, however, prove this in such a general setting of Theorem 2.1. The proof needs some supplementary hypotheses on $V$ and $\mathcal{L}$, and could follow the scheme below.

Since (2.13) holds, the entropy $W[\cdot \mid \cdot]$ dominates the $L^{1}$ metric in which (1.1) generates a dynamical system. To conclude that the (strict) Lyapunov functional $W$ decreases to its unique critical value $0=W\left[u_{\infty} \mid u_{\infty}\right]$ in the set $\left\{z \in L^{1}\left(\mathbb{R}^{d}\right): z \geq 0, \int z=\right.$ $\left.\int u_{0}=\int u_{\infty}\right\}$, we would use the LaSalle invariance principle (see, e.g. [11, Th. 9.2.3 and Th. 9.2.7]). To do that, we need the compactness of the closures of trajectories $\{u(t): t \geq 0\}$ in $L^{1}\left(\mathbb{R}^{d}\right)$. The latter property would follow from their weak compactness 
in $L^{1}\left(\mathbb{R}^{d}\right)$, the property of the uniform space localization of $u(t)$ :

$$
\forall \varepsilon>0 \exists R, T \quad \forall t \geq T \quad \int_{|x| \geq R} u(x, t) d x<\varepsilon,
$$

and the convergence of the norms: $\|u(t)\|_{L^{1}} \rightarrow\left\|u_{\infty}\right\|_{L^{1}}$ which holds because $u(x, t) \geq 0$, $u_{\infty}(x) \geq 0$ and $\int u(x, t) d x=\int u_{\infty}(x) d x$.

The weak $L^{1}\left(\mathbb{R}^{d}\right)$-compactness (characterized by the Dunford-Pettis theorem) is assured by the uniform integrability of $u(t)$ since $W(t) \leq W(0)$ and $\Psi$ in the definition of $W$ is a strictly superlinear function (cf. the de la Vallée-Poussin criterion).

To prove the localization property, we need some additional hypotheses on the mutual relations of $\mathcal{L}$ and $V$.

Note that $\mathcal{I}=0$ in (2.8) characterizes (unique) steady states of (1.1) so that the convergence of $W(t)$ to zero can also be obtained by a standard variational argument $W\left(t_{n}\right) \searrow W_{\infty}, \frac{d W\left(t_{n}\right)}{d t} \rightarrow 0$ for a (and hence any) sequence $t_{n} \nearrow \infty, W_{\infty}=W\left[u_{\infty} \mid u_{\infty}\right]=0$ under the Palais-Smale condition which is, essentially, the $L^{1}\left(\mathbb{R}^{d}\right)$-compactness of the closures of trajectories discussed above. Indeed, if in (2.11) the equality holds, then $\frac{u(x, t)}{u_{\infty}(x)}=\frac{u(y, t)}{u_{\infty}(y)}\left(=1\right.$, by (1.4)) a.e., and $\lim _{t \rightarrow \infty} u(t)=u_{\infty}$ would follow.

We expect that supplementary hypotheses expressing compactness properties of the semigroup generated by $-\mathcal{L}+\nabla \cdot(. \nabla V)$ could guarantee that even a stronger result on the exponential convergence to the steady states in $L^{1}\left(\mathbb{R}^{d}\right)$ will hold, compare the following remark.

REMARK. In the case of a divergence form elliptic second order diffusion operator $\mathcal{L}$ a stronger result is proved (under certain supplementary regularity and size assumptions on $\mathcal{L}$ and $V$ ) in [1]. Namely, the relative entropy $W$ is shown to decay exponentially

$$
W(t) \leq W(0) e^{-\lambda t}
$$

for some $\lambda>0$. The idea is to consider the entropy dissipation relation

$$
\frac{d \mathcal{I}}{d t}=-\mathcal{R}
$$

and to show that $\mathcal{R}$ satisfies

$$
\mathcal{R} \geq \lambda \mathcal{I}
$$

for some $\lambda>0$. This, together with the preliminary result $\lim _{t \rightarrow \infty} W(t)=0$, leads to (2.14). The proof of (2.16) is rather involved and tricky. That proof uses a specific property of divergence form operators $\mathcal{L}=\nabla \cdot(D \nabla)$ with a symmetric positive definite matrix $D=D(x)$ which permitted the authors of [1] to write down the Fokker-Planck equation $u_{t}=\nabla \cdot(D(\nabla u+u \nabla V))$ in an equivalent self-adjoint form

$$
z_{t}=\nabla \cdot(D \nabla z)-z \widetilde{V}
$$

on $L^{2}\left(\mathbb{R}^{d}\right)$ involving the new variable $z=u / \sqrt{u_{\infty}}$ and a new potential $\widetilde{V}$.

The relation (2.16) appears to be equivalent to the generalized logarithmic Sobolev inequality of Bakry-Eméry. Thus, the proof of the exponential convergence of $u(t)$ to $u_{\infty}$ in [1] has given, via (2.16), an alternative proof of this rather delicate extension of the classical Gross logarithmic Sobolev inequality, see [20], [21] for an earlier approach. 
In the framework of [1], the preliminary estimate on the decay of the entropy to 0 is proved using simple spectral theory arguments (and we used the Invariance Principle). On the other hand, the logarithmic Sobolev inequality implies the spectral gap condition for the operator in the form (2.17) of the Fokker-Planck equation, see [1, Sec. 3.2].

We believe that $\mathcal{R}$ satisfies the inequality (2.16) also for general diffusion operators $\mathcal{L}$ and suitable potentials $V$. However, observe that the logarithmic Sobolev inequality for generators of hypercontractive semigroups $e^{-t \mathcal{L}}$ as in [13, Th. 2.2.3] (or in [12, Prop. II.1 and Th. II.5]) is not sufficient to prove (2.16), and thus a proof of (2.16) requires another approach.

\section{Lévy-Fokker-Planck equations and the exponential convergence to their} steady states. The equations of the form

$$
u_{t}+\mathcal{L} u=\nabla \cdot(u x),
$$

called here the Lévy-Fokker-Planck equations, are the main object of study in this section. They are particular examples of (1.1). Moreover, they appear as the rescaled versions of equations

$$
z_{t}+\mathcal{L} z=0
$$

when $\mathcal{L}$ has suitable scaling properties. Indeed, if $\mathcal{L}=(-\Delta)^{\alpha / 2}$, defined by the Fourier multiplier $a(\xi)=|\xi|^{\alpha}, 0<\alpha \leq 2$, corresponds to the $\alpha$-stable law, then the space-time rescaling

$$
z(x, t)=(\alpha t+1)^{-d / \alpha} u\left(x(\alpha t+1)^{-1 / \alpha}, \alpha^{-1} \log (\alpha t+1)\right)
$$

leads exactly to $(3.1)$.

Let $p_{t}^{\alpha}(x-y)=K_{t}(x, y)$ be the integral kernel of the semigroup $e^{-t(-\Delta)^{\alpha / 2}},\left\|p_{t}^{\alpha}\right\|_{L^{q}}=$ $c t^{-d(1-1 / q) / \alpha}$. Thus, the results on the intermediate asymptotics of $z(t)$ (see [6], [9]):

$$
t^{d(1-1 / q) / \alpha}\left\|z(t)-\left(\int z_{0}\right) p_{t}^{\alpha}\right\|_{L^{q}} \rightarrow 0 \text { as } t \rightarrow \infty
$$

are equivalent to the results on the convergence of $u(t)$ to $u_{\infty}$.

To the best of our knowledge, [10] was the first result on the asymptotic resemblance of $z(t)$ to a multiple of the heat kernel $p_{t}^{2}$, preceding the fine analysis of the self-similar $L^{1}$ large time behavior of solutions of the heat equation, i.e. (3.2) with $\mathcal{L}=-\Delta$ (and more general parabolic equations). They used in [10] arguments involving estimates for fundamental solutions of considered equations to show that if $z_{0} \in L^{1}, \int z_{0}=0$, then $\|z(t)\|_{L^{1}} \rightarrow 0$ as $t \rightarrow \infty$, but they do not get estimates for the decay rate. After then, several papers appeared, and we mention only [19] employing entropy methods, together with very general, technical results in [14] applicable in a much wider context. An application of these ideas to nonlinear problems can be found in [6], [8], [9].

Let us consider the equation (3.1) where $\mathcal{L}$ is a Lévy diffusion generator defined by its symbol $a(\xi)$ as in the Lévy-Khinchin formula $(3.143)$ in $[15]: \widehat{\mathcal{L} u(\xi)}=a(\xi) \widehat{u}(\xi)$. Moreover, we suppose, as in [8] that

$$
0<\liminf _{\xi \rightarrow 0} \frac{a(\xi)}{|\xi|^{\alpha}} \leq \limsup _{\xi \rightarrow 0} \frac{a(\xi)}{|\xi|^{\alpha}}<\infty,
$$




$$
0<\inf \frac{a(\xi)}{|\xi|^{2}}
$$

are satisfied for some $\alpha$ (which must belong to $(0,2]$ ), as well as $a$ is sufficiently smooth for $\xi \neq 0$.

REMARK. Our assumptions on the symbol $a$ guarantee not only the hyper- and ultracontractivity of the semigroup $e^{-t \mathcal{L}}$, cf. $[8,(2.6)]$, where the decay estimates

$$
\left\|e^{-t \mathcal{L}} v\right\|_{L^{p}} \leq C \min \left(t^{-d(1-1 / p) / 2}, t^{-d(1-1 / p) / \alpha}\right)\|v\|_{L^{1}},
$$

for the semigroup $e^{-t \mathcal{L}}$ are discussed, but also the boundedness of the solutions $u \mapsto u(t)$ of (3.1) from $L^{1}\left(\mathbb{R}^{d}\right)$ to $L^{p}\left(\mathbb{R}^{d}\right)$. Note that a similar boundedness result with $u_{0} \in L^{p}$ follows using the entropy $\Psi_{p}$ with any $p>1$ as in Theorem 2.1. If $V$ is such that $\sup _{x \in \mathbb{R}^{d}} \Delta V(x)<\infty$, then $\lim \sup _{t \rightarrow \infty}\|u(t)\|_{L^{p}}<\infty$ for each $u_{0} \in\left(L^{1} \cap L^{p}\right)\left(\mathbb{R}^{d}\right)$. To see this, observe that

$$
\frac{1}{p} \frac{d}{d t}\|u\|_{L^{p}}^{p}+\int \mathcal{L} u|u|^{p-2} u=-\int u \nabla V \cdot \nabla\left(|u|^{p-2} u\right)=\frac{p-1}{p} \int \Delta V|u|^{p} .
$$

Using a result extending the classical Nash inequality which relates the decay of the semigroup $e^{-t \mathcal{L}}$ and the integrals $\int \mathcal{L} u v$ (cf. [13, (2.2.7) for $p \geq 2$, Th. 2.4.6], and [12, Prop. II.1, Th. II.5] for a more general announcement, or [23, the proof of Prop. II. 5.4] where the following extension of the Kato-Beurling-Deny inequality

$$
\frac{4(p-1)}{p^{2}} \int\left(\mathcal{L}|u|^{p / 2}\right)|u|^{p / 2} \leq \int(\mathcal{L} u)|u|^{p-1} \operatorname{sgn} u
$$

is shown), we get

$$
\frac{d}{d t} w+c w^{1+\varepsilon} \leq C w
$$

where $w(t)=\|u(t)\|_{L^{p}}^{p}$, and $\varepsilon=1 /((p-1) \alpha)$. We refer the readers for details of the use of the Kato-Beurling-Deny inequality to [8]. The differential inequality (3.6) leads to

$$
\log w-\varepsilon^{-1} \log \left(C-c w^{\varepsilon}\right) \leq C t,
$$

hence $w^{\varepsilon}(t) \leq C e^{\varepsilon C t}\left(1+c e^{\varepsilon C t}\right)^{-1}$, and $\lim \sup _{t \rightarrow \infty} w^{\varepsilon}(t) \leq C$ follows. A similar argument applied to $u(t)-u_{\infty}$ shows (local) asymptotic stability of $u_{\infty}$.

A more precise statement in Theorem 3.1 below is proved using different arguments of spectral theory.

It is convenient to study the equation (3.1) in the Fourier variables where this takes the form

$$
v_{t}+a(\xi) v+\xi \cdot \nabla v=0
$$

with $v=\widehat{u}$ and $\nabla=\nabla_{\xi}$. The initial condition (1.2) leads to

$$
v_{0}(\xi)=\widehat{u_{0}}(\xi)
$$

THEOREM 3.1. The solutions of the equation (3.1) converge to the corresponding steady states at an exponential rate

$$
\left\|u(t)-u_{\infty}\right\|_{L^{p}} \leq C e^{-\varepsilon t}
$$

for all $2 \leq p \leq \infty$, some $\varepsilon=\varepsilon(p)>0$, and $C$ which depend on $\mathcal{L}, p$, and $u_{0}$, respectively. 
COROLlaRY 3.1. Under some more restrictive regularity and decay assumptions on $u_{0}$, the exponential convergence

$$
\left\|u(t)-u_{\infty}\right\|_{L^{p}} \leq C e^{-\varepsilon t}
$$

holds for all $1 \leq p \leq \infty$.

Proof. The scheme of the proof of this corollary is fairly standard. It suffices to note that

$$
\|u\|_{L^{1}} \leq C\|\widehat{u}\|_{L^{2}}^{1-d /(2 m)}\left\|D^{m} \widehat{u}\right\|_{L^{2}}^{d /(2 m)}
$$

for every integer $m>d / 2$, e.g. $m=[d / 2]+1$, and a constant $C=C(d, m)$. This is a well known fact, frequently used in harmonic analysis. We refer the reader for an elementary proof of (3.11) to [5, Example 2, p. 1748].

Indeed, modulo assumptions on the regularity of the symbol $a$ off the origin and on $v_{0}=\widehat{u_{0}}$ (hence on the moments of $\left.u_{0}\right)$, the solution $\left(D^{m} v-D^{m} v_{\infty}\right)$ of the linear equation (3.1) decays exponentially to 0 in $L_{\xi}^{2}\left(\mathbb{R}^{d}\right)$. We will not pursue here the question of optimal assumptions on $a$ and $u_{0}$ needed for the above reasoning.

Once the exponential convergence of $\left\|u(t)-u_{\infty}\right\|_{L^{1}}$ has been obtained, the convergence of $\left\|u(t)-u_{\infty}\right\|_{L^{p}}$, again at an exponential rate, follows by an interpolation argument and $(3.9)$.

Proof of Theorem 3.1. The Fourier transform $v_{\infty}$ of the steady state $u_{\infty}$ satisfies the (first order partial differential) equation

$$
\xi \cdot \nabla v_{\infty}+a(\xi) v_{\infty}=0
$$

Thus, we have a unique steady state corresponding to mass 1

$$
v_{\infty}(\xi)=\exp (-A(\xi))
$$

where

$$
A(\xi)=\int_{0}^{1} a(s \xi) s^{-1} d s .
$$

and $\int v_{\infty}(\xi) d \xi \equiv c_{\mathcal{L}, V}$. Note that $A(\xi)$ is well defined since $a(0)=0,0 \leq a(\xi) \leq c|\xi|^{\alpha}$ for small $|\xi|$ and some $0<\alpha \leq 2$. Moreover, if $a(\xi) \sim|\xi|^{\alpha}$ for $|\xi| \searrow 0$, then also $A(\xi) \sim|\xi|^{\alpha}$, and $A(\xi) \rightarrow \infty$ together with $a(\xi) \rightarrow \infty$ and/or $|\xi| \rightarrow \infty$. The fact that $A$ is a Lévy symbol easily follows from, e.g., the Schoenberg theorem [15, Th. 3.6.11]. In particular, if $a(\xi)=|\xi|^{\alpha}$, then $u_{\infty}=p_{1}^{\alpha}$.

Rewriting the equation (3.1) as

$$
\exp (A(\xi)) v_{t}+\xi \cdot \nabla(\exp (A(\xi)) v)=0
$$

we arrive at the following Fourier transform of the solution of (3.1)

$$
v(\xi, t)=\exp \left(A\left(e^{-t} \xi\right)\right) v_{0}\left(e^{-t} \xi\right) \exp (-A(\xi)) .
$$

Now, we will prove that there exists an $\varepsilon>0$ such that

$$
\int \Psi_{2}\left(\frac{v(t)}{v_{\infty}}\right) v_{\infty} \leq C e^{-2 \varepsilon t}
$$


Since $A(\xi) \geq 0$, it follows that $v_{\infty}^{-1}(\xi)=\exp (A(\xi)) \geq 1$. Hence, the $\Psi_{2}$ entropy controls the $L^{2}$ norm

$$
\int \Psi_{2}\left(\frac{v(t)}{v_{\infty}}\right) v_{\infty} \geq \int\left(v(t)-v_{\infty}\right)^{2}
$$

and therefore

$$
\left\|v(t)-v_{\infty}\right\|_{L^{2}}=\left\|\exp (-A(\xi))\left(\exp \left(A\left(e^{-t} \xi\right)\right) v_{0}\left(e^{-t} \xi\right)-v_{0}(0)\right)\right\|_{L^{2}} \leq C e^{-\varepsilon t} .
$$

The latter is clearly equivalent to the statement on the exponential convergence of $u(t)$ to $u_{\infty}$ in $L^{2}$.

To get (3.16) we show that for each $0<\varepsilon<\alpha$

$$
\mathcal{J} \equiv e^{2 \varepsilon t}\left\|\exp \left(-\frac{1}{2} A(\xi)\right)\left(\exp \left(A\left(e^{-t} \xi\right)\right) v_{0}\left(e^{-t} \xi\right)-v_{0}(0)\right)\right\|_{L^{2}}^{2}
$$

is bounded as $t \rightarrow \infty$. Given $\delta>0$ we split the quantity $\mathcal{J}$ into two integrals $\mathcal{J}=J_{1}+J_{2}$ of the function

$$
\exp (-A(\xi))\left(\exp \left(A\left(e^{-t} \xi\right)\right) v_{0}\left(e^{-t} \xi\right)-v_{0}(0)\right)^{2}
$$

over $\left\{0 \leq|\xi|<\delta e^{t}\right\}$ and $\left\{\delta e^{t} \leq|\xi|\right\}$, resp. Observe that by $u_{0} \geq 0$, we have $\nabla v_{0}(0)=0$, hence

$$
\left|\exp (A(\eta)) v_{0}(\eta)-v_{0}(0)\right| \leq C A(\eta) \leq C|\eta|^{\alpha}
$$

for all $|\eta|<\delta$ with $\delta>0$ small enough (in fact $\delta=\delta\left(v_{0}\right)=\delta\left(\left\|u_{0}\right\|_{L^{1}}\right)$ ), and $C=$ $C\left(v_{0}(0)\right)=C\left(\left\|u_{0}\right\|_{L^{1}}\right)$. Therefore

$$
J_{1} \leq \int_{|\xi|<\delta e^{t}} \exp (-A(\xi))|\xi|^{2 \alpha} e^{(2 \varepsilon-2 \alpha) t} d \xi \rightarrow 0
$$

holds as $t \rightarrow \infty$. To estimate the integral $J_{2}$ let us note that under the assumptions on the symbol $a$ we have for each $b>0$ and all $t \geq T=T(b)$

$$
A(\xi) \geq b t A\left(e^{-t} \xi\right) \text {. }
$$

Here we take $b>\alpha(4 \inf \{A(\eta):|\eta| \geq \delta\})^{-1}$. Therefore (3.18) leads to the bound

$$
\begin{aligned}
& J_{2} \leq C \int_{\delta e^{t} \leq|\xi|} \exp \left(\left(2 \varepsilon-b A\left(e^{-t} \xi\right)\right) t\right. \exp \left(2 A\left(e^{-t} \xi\right)\right) d \xi \\
& \leq C \int_{\delta e^{t} \leq|\xi|} \exp \left(-A\left(e^{-t} \xi\right)\right) d \xi<\infty
\end{aligned}
$$

for $t \geq \max \{T(b), 6 / b\}$ because $(b t-3) A(\eta) \geq \frac{1}{2} b t A(\eta) \geq 2 \varepsilon t$ holds.

Now, using the Csiszár-Kullback inequalities for $W$ with $\Psi_{2}$ and [1, Lemma 2.7] on the comparison of admissible entropies with $\Psi_{1}$ and $\Psi_{2}$, we obtain the exponential convergence to 0 of the norms $\left\|v(t)-v_{\infty}\right\|_{L^{p}}$ with $1 \leq p \leq 2$. Thus, the exponential decay of $\left\|u(t)-u_{\infty}\right\|_{L^{q}}$ for $2 \leq q \leq \infty$ follows by the Hausdorff-Young inequality.

Finally, let us remark that, contrarily to the spectral theory approach in Section 3, the entropy method is robust and can be extended to certain nonlinear problems, cf. [2], [3] and [4]. 
Acknowledgements. The authors wish to thank Peter Markowich for sharing his ideas concerning various generalizations of Fokker-Planck equations, Wojbor Woyczyński for discussions on probabilistic interpretations of those equations, and Jean Dolbeault for interesting comments. Grant support from KBN 50/P03/2000/18 is gratefully acknowledged as well as ÖAD-KBN Austrian-Polish Scientific-Technical Collaboration Agreement which made possible authors' visits to the Erwin Schrödinger Institute in Vienna. Last but not least, we heartily thank Dariusz Wrzosek and an anonymous referee for the constructive criticism.

\section{References}

[1] A. Arnold, P. A. Markowich, G. Toscani, A. Unterreiter, On convex Sobolev inequalities and the rate of convergence to equilibrium for Fokker-Planck type equations, Comm. Partial Differential Equations 26 (2001), 43-100.

[2] P. Biler, J. Dolbeault, Long time behavior of solutions of Nernst-Planck and DebyeHückel drift-diffusion systems, Ann. Henri Poincaré 1 (2000), 461-472.

[3] P. Biler, J. Dolbeault, M. J. Esteban, G. Karch, Stationary solutions, intermediate asymptotics and large time behaviour of type II Streater's models, Adv. Differential Equations 6 (2001), 461-480.

[4] P. Biler, J. Dolbeault, P. A. Markowich, Large time asymptotics of nonlinear drift-diffusion systems with Poisson coupling, Transport Theory Statist. Phys. 30 (2001), 521-536.

[5] P. Biler, J. Dziubański, W. Hebisch, Scattering of small solutions to generalized Benjamin-Bona-Mahony equation in several space dimensions, Comm. Partial Differential Equations 17 (1992), 1737-1758.

[6] P. Biler, G. Karch, W. A. Woyczyński, Asymptotics for multifractal conservation laws, Studia Math. 135 (1999), 231-252.

[7] P. Biler, G. Karch, W. A. Woyczyński, Multifractal and Lévy conservation laws, C. R. Acad. Sci. Paris Sér. I Math. 330 (2000), 343-348.

[8] P. Biler, G. Karch, W. A. Woyczyński, Asymptotics for conservation laws involving Lévy diffusion generators, Studia Math. 148 (2001), 171-192.

[9] P. Biler, G. Karch, W. A. Woyczyński, Critical nonlinearity exponent and selfsimilar asymptotics for Lévy conservation laws, Ann. Inst. H. Poincaré Anal. Non Linéaire 18 (2001), 613-637.

[10] Z. Brzeźniak, B. Szafirski, Asymptotic behaviour of $L^{1}$ norms of solutions to parabolic equations, Bull. Pol. Acad. Sci. Ser. Math. 39 (1991), 1-10.

[11] Th. Cazenave, A. Haraux, An Introduction to Semilinear Evolution Equations, Oxford Lecture Ser. Math. Appl. 13, Oxford University Press, New York, 1998.

[12] T. Coulhon, Ultracontractivity and Nash type inequalities, J. Funct. Anal. 141 (1996), $510-539$.

[13] E. B. Davies, Heat Kernels and Spectral Theory, Cambridge Tracts in Math. 92, Cambridge University Press, Cambridge, 1989.

[14] J. Duonndikoetxea, E. Zunzua, Moments, masses de Dirac et décomposition de fonctions, C. R. Acad. Sci. Paris Sér. I Math. 315 (1992), 693-698.

[15] N. Jасов, Pseudodifferential Operators and Markov Processes, Vol. I: Fourier Analysis and Semigroups, Imperial College Press, London, 2001.

[16] M. Krasnoselskit, Ya. Rutickit, Convex Functions and Orlicz Spaces, Noordhoff, Groningen, 1961. 
[17] D. Schertzer, M. Larchevêque, J. Duan, V. V. Yanovsky, S. Lovejoy, Fractional Fokker-Planck equation for nonlinear stochastic differential equations driven by nonGaussian Lévy stable noises, J. Math. Phys. 42 (2001), 200-212.

[18] M. SHIIno, Free energies based on generalized entropies and H-theorems for nonlinear Fokker-Planck equations, J. Math. Phys. 42 (2001), 2540-2553.

[19] G. Toscani, Kinetic approach to the asymptotic behaviour of the solutions to diffusion equations, Rend. Mat. Appl. (7) 16 (1996), 329-346.

[20] G. Toscani, Sur l'inégalité logarithmique de Sobolev, C. R. Acad. Sci. Paris Sér. I Math. 324 (1997), 689-694.

[21] G. Toscani, Entropy production and the rate of convergence to equilibrium for the FokkerPlanck equation, Quart. Appl. Math. 57 (1999), 521-541.

[22] A. Unterreiter, A. Arnold, P. Markowich, G. Toscani, On generalized CsiszárKullback inequalities, Monatsh. Math. 131 (2000), 235-253.

[23] N. Th. Varopoulos, L. Saloff-Coste, T. Coulhon, Analysis and Geometry on Groups, Cambridge Tracts in Math. 100, Cambridge University Press, Cambridge, 1992. 